Patient and family denied knowledge of intentional or accidental ingestion. Police investigation was inconclusive.

Lessons to be Learned Larger supplies of fomepizole are now kept in stock within Health Board. Supplies were missing from emergency cupboards when stock was needed, despite being on stock lists, necessitating courier fees to transfer stock from elsewhere. Procedures reviewed to ensure that stock is available in emergency cupboards at all times.

This patient demonstrated that current (target) stock levels of fomepizole were inadequate for providing treatment during CRRT as the required doses are substantially higher (administered every four hours rather than every twelve hours) and would have lasted less than 12 hours for this average sized teenager. National Rarely Used Medicines list was updated to reflect actual stock levels and other hospitals increased their stockholding due to the realisation that existing stock was inadequate and that further supplies were hard to obtain out of working hours.

\section{SP3 EVALUATING THE IMPACT OF CONCENTRATED STANDARDISED PARENTERAL NUTRITION ON GROWTH OF PRETERM INFANTS}

Lucy Stachow*, T'ng Chang Kwok, Ramune Snuggs, Rowan Toyer, Emmanuel Oyewole, Deepa Panjwani, Kamil Effendi. University Hospitals of Leicester NHS Trust

\subsection{6/archdischild-2020-NPPG.3}

Background and Aim Concentrated standardised parenteral nutrition (CSPN) may reduce the delay in commencing parenteral nutrition (PN) in preterm infants compared with conventional individualised PN. Optimisation of early nutrition, with emphasis on earlier commencement of PN to include amino acids and addition of lipids within 24 hours of birth, ameliorates early postnatal growth failure. ${ }^{1} 2$ Cumulative nutritional deficit often seen in significantly preterm infants may lead to poor neurodevelopmental outcome. ${ }^{3} 4$ CSPN was introduced in our neonatal unit in December 2017 with the objective of improving early nutrition. The aim of this service evaluation was to assess the suitability of CSPN and its impact on the growth of preterm infants in our tertiary level neonatal unit.

Methods In December 2017, the neonatal PN provided was switched from individualised PN to CSPN based on a modified 'SCAMP' regimen. Retrospective and prospective growth parameter data was collected for infants receiving PN within 24 hours of birth born between September to November 2017 (individualised PN arm) and from September to November 2018 (CSPN arm). Infants were excluded if they died or transferred out of the local neonatal service before day 28 of life, or died before transitioning from PN to full enteral feeds. Weight and head circumference at birth, 28 days old and 36 weeks corrected gestation/discharge were converted to $\mathrm{z}$ scores using the LMS method. The Mann-Whitney test was used to compare continuous data. Annual PN expenditure, and wastage of ordered PN, before and after the switch to CSPN, was calculated using the pharmacy stock management system, pharmacist and finance records.

Results 20 infants (mean gestational age 28 weeks) and 21 infants (mean gestational age 29.6 weeks) were included in the CSPN and individualised PN groups respectively. There were no differences in demographic data of each group. CSPN was commenced earlier (median 8 hours old $(n=20)$ ) than individualised PN (median 25 hours old $(n=19)$ ), $(U=42$, $\mathrm{p}<0.0001)$. There was no statistical difference in the change in weight $\mathrm{z}$ score from birth at 28 days old (median -0.47 $(n=20)$ CSPN vs $-0.66(n=19)$ individualised $P N, U=178.5$, $\mathrm{p}=0.75)$ and at 36 weeks corrected gestation/discharge (median $-0.72(n=20)$ CSPN vs $-0.86 \quad(n=21)$ individualised $\mathrm{PN}, \mathrm{U}=106, \mathrm{p}=0.7)$. There was insufficient data collected to analyse effect on head circumference. Replacing individualised PN with CSPN resulted in a $37 \%$ reduction in procurement costs, despite an increase in the wastage of ordered PN from $7.2 \%$ to $8.5 \%$.

Conclusion A PN strategy using concentrated standardised PN can be implemented successfully in a tertiary neonatal unit setting in the United Kingdom and allows earlier commencement of PN. Use of CSPN appeared to have no adverse effect on weight gain, although small sample size may account for the lack of statistical significance in improvement of weight $\mathrm{z}$ score seen. Improved rates of head circumference documentation for our patients are required. Introducing CSPN resulted in a considerable reduction in procurement costs, and identifying strategies to minimise wastage of CSPN bags would further improve cost-effectiveness.

\section{REFERENCES}

1. Morgan C, McGowan P, Herwitker $S$, et al. Postnatal head growth in preterm infants: a randomised controlled parenteral nutrition study. Pediatrics 2014;133: e120-8.

2. Moyses $H E$, Johnson MJ, Leaf $A A$, et al. Early parenteral nutrition and growth outcomes in preterm infants: a systematic review and meta-analysis. Am J Clin Nutr 2013;97:816-26.

3. Ehrenkranz RA, Dusick AM, Vohr BR, et al. Growth in the neonatal intensive care unit influences neurodevelopmental and growth outcomes of extremely low birth weight infants. Pediatrics 2006;117:1253-61.

4. Dusick AM, Poindexter BB, Ehrenkranz RA, et al. Growth failure in the preterm infant: can we catch up? Semin Perinatol 2003;27:302-10.

\section{SP4 MEDICINES OPTIMISATION IN ACTION: THE DEVELOPMENT OF A DOSING SCHEDULE AND AN EXTEMPORANEOUS FORMULATION OF PAZOPANIB TO TREAT A CHILD WITH A RECURRENT DESMOID TUMOUR OF THE HEAD AND NECK}

Victoria Holden*, Andrew Lowey, Simone Wilkins, Rachel Greer. Leeds Teaching Hospitals

\subsection{6/archdischild-2020-NPPG.4}

Background International experts recommended pazopanib to treat a 9 year old boy with a recurrent desmoid tumour. The tumour had progressed, leading to blockage of the airway and dysphagia. The child had previously received three lines of intravenous chemotherapy as well as surgery and local radiotherapy. Treatment intent was to give an effective oral therapy with minimal side effects aiming to maintain a reasonable quality of life and prevent further life threatening respiratory compromise. As the child had an unsafe swallow, the drug would need to be given via a gastrostomy tube.

Pharmacy Input A literature review was completed to establish the evidence to support the use and dose of pazopanib to treat desmoid type tumours in children. ${ }^{1}{ }^{2}$ Attempts made to find a commercial supply of pazopanib liquid yielded no results. In the absence of any data or experience from other principal children's cancer centres in the UK, first principles were used to review the physico-chemical properties of the molecule. Given the highly insoluble nature of pazopanib, an extemporaneous formulation for a pazopanib suspension was developed using Orasweet ${ }^{\circledR}$, by adapting a formula suggested by the University of Oklahoma. ${ }^{3}$ A risk assessment for the 
preparation process was completed and a mini isolator was recommissioned for this purpose following appropriate testing by Quality Control. The Department of Pharmacology at Newcastle University were contacted to establish if therapeutic drug level analysis might be possible. Local approval for pazopanib use was obtained from the Drug and Therapeutic Group and an IFR was submitted to NHS England.

Challenges It was challenging to establish a dose regimen since the recommended paediatric dose from Phase I studies of pazopanib were dependent on the formulation used. ${ }^{1}{ }^{4}$ Pharmacokinetic sampling was not possible as no assay had been developed in the UK. The lack of availability of any commercial or compassionate use liquid preparation meant that the only way of giving the drug to this child was to prepare an extemporaneous preparation, despite paucity of evidence to support the stability of the preparation. Funding was not approved by NHS England; local funding was agreed.

Outcome and Discussion The child commenced treatment with the suspension in October 2018, administered via the gastrostomy tube. By July 2019 the patient had completed 10 cycles of therapy. Treatment was well tolerated. Minor side effects included abdominal and leg pain, vomiting, and a change in hair colour. The patient has had a good clinical response and a recent scan has shown substantial improvements in morphological appearance and size of the tumour.

These results indicate that a locally prepared extemporaneous oral chemotherapy suspension can be successfully used to deliver treatment for a rare type of children's cancer. Pharmacy colleagues from across the department collaborated to facilitate this novel treatment option.

\section{REFERENCES}

1. Glade Bender J, Lee A, Reid J, et al. Phase I pharmacokinetic and pharmacodynamic study of pazopanib in children with soft tissue sarcoma and other refractory solid tumors: a children's oncology group phase I consortium report. JCO 2011;31:3034-3043.

2. Agresta $L$, Lee $H$, Turpin $B$, et al. Pazopanib therapy for desmoid tumors in adolescent and young adult patients. Pediatr Blood Cancer 2018;65:e26968.

3. Allen L Jr. Pazopanib $50 \mathrm{mg} / \mathrm{ml}$ oral suspension. US Pharm 2015;40:61-62.

4. Heath E, Forman $\mathrm{K}$, Malburg L, et al. A phase I pharmacokinetic and safety evaluation of oral pazopanib dosing administered as crushed tablet or oral suspension in patients with advanced solid tumors. Invest New Drugs 2012;30:1566-1574.

\section{\begin{tabular}{l|l} 
SP5 & TENFOLD MEDICATION ERRORS IN CHILDREN - WELSH
\end{tabular} PAEDIATRIC SURVEILLANCE UNIT STUDY 2017-9}

${ }^{1}$ Yincent Tse*, ${ }^{2}$ David Tuthill. 'Great North Children's Hospital, Newcastle Upon Tyne; ${ }^{2}$ Children's Hospital for UK

10.1136/archdischild-2020-NPPG.5

Aims To establish the incidence and characteristics of tenfold or greater and a tenth or less medication errors in children $<16$ years in Wales to help inform patient safety on a population level.

Method Population-based incidence study in Wales, UK, from June 2017 - May 2019 (24 months). Cases were reported from paediatricians and hospital pharmacists using the monthly Welsh Paediatric Surveillance Unit (WPSU).

Results 46 confirmed incidents in 44 children from 63 notifications were identified. Cases came from 8 hospitals in Wales with $29(63 \%)$ from the sole tertiary hospital. Median age was 1.7 (range 1 week to 15 ) years and weight $10 \mathrm{~kg}(0.6$ to 59$)$.

$39(85 \%)$ were overdosing (up to $1000 \mathrm{x}$ fold error) and 7 underdosing. 40 different medications were involved, 16
(37\%) intravenous. Of 29 cases involving enteral medication, 26 (90\%) were liquid formulations. Three cases were discharge medication prescribed or dispensed incorrectly and administrated at home. Stage of errors were primarily in prescribing $37(80 \%)$, administration $7(16 \%)$ and dispensing 2 (4\%).

$18(42 \%)$ cases reached the patient, 10 from prescribing. Seven cases were spotted after multiple doses were given. Six errors resulted in harm, three which required intensive care treatment. No deaths or permanent disabilities were reported. Half $(23 / 46)$ of all errors reported and two-thirds (12/18) of cases that reached the child occurred in $<10 \mathrm{~kg}$ children.

Several human factor themes were identified: Prescribing confusion between gram milligram and microgram (none reached patient, $n=7)$, confusing between $\mathrm{mg}$ and $\mathrm{mg} / \mathrm{kg}(\mathrm{n}=6$ including 3 underdosing errors), leading zero errors (e.g. 0.1 vs $0.001 \mathrm{mg}, \mathrm{n}=6$ ) and prescribing reconciliation errors where admitting doctor attempted to prescribe chronic medication in $\mathrm{mg}$ by reversing calculating liquid dosage expressed in $\mathrm{mL}$ $(n=4)$.

During this study period 164,000 hospital admissions occurred in children $<16$ years in Wales. Our data estimates a tenfold error incidence of 1:3600 paediatric admissions, with drug reaching the child in 1:9000 admissions.

Conclusion In this unique first ever population surveillance study, tenfold errors in children occurred at every stage of medication process and in the full range of care settings. Errors found were very different from those obtained from tertiary hospital single centre study and UK National Reporting and Learning System (NRLS). Strategies for error reduction will be more productive if designed across a whole national healthcare system.

\section{\begin{tabular}{|l|l}
\hline SP6 & BUILDING ON THE DRUGGLE: PERSONALISED
\end{tabular} FEEDBACK TO IMPROVE AND MAINTAIN GOOD PRESCRIBING PRACTICE}

Thomas Wyllie*. University Hospital UK

\subsection{6/archdischild-2020-NPPG.6}

Background Inspired by work from a number of other centres, ${ }^{1} 2$ a weekly 'Druggle' was set up on our 28 cot tertiary, level 3 neonatal intensive care unit in June 2018. The Druggle is a short pharmacist-led briefing in the clinical area involving doctors and nurses, focussing on prescribing and administration issues and errors. Over the first year a concurrent zero tolerance audit shows an improvement in prescribing practice, with an increased number of charts with zero errors (63\% in June 2018, 95\% in June 2019). Despite the improvements in prescribing practice, average attendance at the Druggle has fallen from 17 people per week to 7 over the year. It was decided to consider personalised feedback on prescribing as a potential new mechanism to improve and maintain prescribing standards.

Aim To investigate if structured, personalised feedback to prescribers on a neonatal unit could be an innovative way of improving prescribing standards and patient safety. The project was set up to gain an insight into prescribers attitudes towards prescribing feedback and to see what impact that feedback might have on their attitudes after it had been carried out.

Method All prescribers on the unit were invited to complete an online questionnaire which included questions on previous 